\title{
Modelo de enseñanza para la atención inclusiva a las necesidades no asociadas a una discapacidad de los estudiantes de la Unidad Educativa Ciudad de Valencia
}

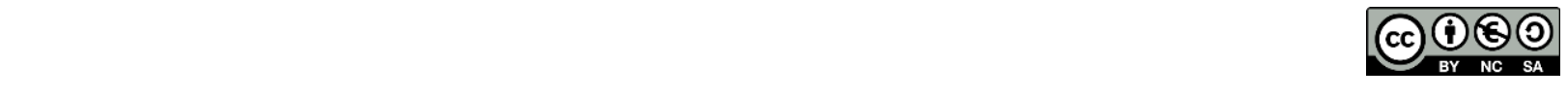

Teaching model for inclusive attention to the needs not associated with a disability of the students of the City of Valencia Educational Unit

Silvia Leonor García Parrales. ${ }^{1}$, Carlos Mego Cubas. ${ }^{2}$ \& Gabriel Arturo Pazmiño Solys. ${ }^{3}$

Recibido: 21-06-2020 /Aceptado: 20-07-2020 / Publicado: 07-08-2020

\begin{abstract}
.
DOI: $\underline{\text { https://doi.org/10.33262/concienciadigital.v3i3.1.1377 }}$

Introduction: In Ecuador the issue of inclusion is something that has occurred recently, since in previous times the term integration was mentioned, which at first glance seems to mean the same thing, but it is not. The Ecuadorian State has made significant changes and invested in education, so that it becomes of quality and warmth. Objective: To include boys and girls with special educational needs in regular classrooms at the Ciudad de Valencia Educational Unit of the Cantón Valencia. Methodology. The research design was quantitative, applied, field, action, retrospective and descriptive. The population was students with special abilities from the Valencia City Educational Unit of the Canton Valencia. Results. In the teaching work, the socio-affective relationship of the students must be taken into account, instilling values of respect among them, and that in turn must be immersed in the content taught in classes. It is important that legal representatives and parents of families should be more aware of the activities carried out by their representatives, and get involved in programs carried out by the school to learn a little more about inclusive
\end{abstract}

\footnotetext{
${ }^{1}$ Magister en Gestión de la Educación. Aspirante al grado de Doctor en Educación PhD. Unidad Educativa Ciudad de Valencia. Provincia de los Ríos. Ecuador., silvialeogarciap@hotmail.com, silvia.garcia@educacion.gob.ec

2Doctor. Universidad Cesar Vallejo. Piura. Perú., mcegoc_@hotmail.com

${ }^{3}$ Doctor en Ingeniería Industrial. PhD. Master en Gestión de Proyectos Sociales y Productivos. Gaps Representaciones., gapsrio@gmail.com
} 
education, and understand that it is not a risk to integrate to boys and girls with special educational needs in the classroom.

Keywords: Model, teaching attention, education, disability.

\section{Resumen}

Introducción: En Ecuador el tema de inclusión es algo que se ha dado recientemente, puesto que en tiempos anteriores se mencionaba el término integración que a simple vista parece significar lo mismo, pero no es así. El Estado ecuatoriano ha realizado cambios significativos y ha invertido en la educación, para que esta se vuelva de calidad y calidez. Objetivo: Incluir a niños y niñas con necesidades educativas especiales en las aulas regulares en la Unidad Educativa Ciudad de Valencia del Cantón Valencia. Metodología. El diseño de investigación fue cuantitativo, de tipo aplicada, de campo, de acción, retrospectiva y descriptiva. La población fue los estudiantes con habilidades especiales de la Unidad Educativa Ciudad de Valencia del Cantón Valencia.. Resultados. En la labor docente se deben tomar en cuenta la relación socio-afectiva de los estudiantes, inculcar valores de respeto entre ellos, y que a su vez deben estar inmersos en los contenidos que imparte en clases. Es importante que los representantes legales y padres de familias, deben estar más al tanto de las actividades que realizan sus representados, e involucrarse en programas que realiza la escuela para conocer un poco más de la educación inclusiva, y comprender que no es ningún riesgo integrar a niños y niñas con necesidades educativas especiales en el aula.

Palabras claves: Modelo, atención enseñanza, educación, discapacidad.

\section{Introducción}

Con el Modelo Social y de Derechos a la Discapacidad, resulta evidente que está interrelacionado e impulsado por los acontecimientos socio-políticos, culturales y económicos que suceden en cada época histórica. De este modo el problema de la discapacidad no está en el individuo sino en la sociedad que lo rodea, Este movimiento multidimensional nace dentro de la disciplina de las ciencias sociales, el análisis de las políticas sociales y la lucha por los derechos civiles; específicamente aquellos relacionados con los derechos de las personas con discapacidad.

Las aportaciones del sociólogo Pierre Bourdieu (2007) [1998], 1991; Bourdieu y Wacquant, 1995), y de las revisiones de las lecturas de la investigación de Soledad Torres Dávila (2004). Asumirla en este sentido significa concebir la discapacidad, en tanto que campo científico y político, en un sentido relacional. 
La discapacidad promueve diversos poderes desde los sujetos, las prácticas y los discursos, que pueden buscar mantener el campo con su dinámica propia, transformarlo o simplemente resistirlo. Sin embargo, estas estrategias están condicionadas por las percepciones que los sujetos tienen sobre la discapacidad como campo y que a su vez las define, da forma y contenido a sus prácticas y discursos (Torres Dávila, 2004).

En el caso de las personas con discapacidad, la ideología de la normalidad no sólo las define por lo que no tienen: su falta, su déficit, su desviación, su ausencia y su carencia, sino que también y simultáneamente confirma el "estar completo" de las no discapacitadas, que suelen ser igualadas y asignadas a la categoría de normales. Esta lógica binaria (normal/ a-normal) se asienta sobre el convencimiento del valor de la normalidad: está bien "ser normal" y, si alguien no lo es, le resulta imperativo hacer los tratamientos de rehabilitación necesarios para acercarse lo más posible a ese estado o esa condición (Balza, 2011).

En la labor docente se deben tomar en cuenta la relación socio-afectiva de los estudiantes, inculcar valores de respeto entre ellos, y que a su vez deben estar inmersos en los contenidos que imparte en clases.

Es importante que los representantes legales y padres de familias, deben estar más al tanto de las actividades que realizan sus representados, e involucrarse en programas que realiza la escuela para conocer un poco más de la educación inclusiva, y comprender que no es ningún riesgo integrar a niños y niñas con necesidades educativas especiales en el aula.

Los estudiantes por su parte deben exigir a su docente cambiar la metodología de trabajo e innovar en la creatividad de recursos didácticos llamativos que faciliten el aprendizaje y promuevan la integración de todos.

Las autoridades deben gestionar actividades de formación docente con relación a la educación inclusiva, para que ellos pueden realizar las adaptaciones pertinentes en el aula de clases.

La inclusión a las necesidades no asociadas a una discapacidad es un tema de discusión crítica con el fin de no estigmatizar a niños niñas adolescentes, las necesidades que no se asocian a una discapacidad no se pueden ser detectar de manera visual pero que si se puede trabajar 
en base a técnicas e instrumentos que vayan dirigidos a esa población de estudio vulnerable, no obstante el docente debe estar capacitado preparado en una formación contantes pero con un compromiso fundamentado al cambio social, enfocado en los principios y valores que le permitan mantener un excelente desempeño.

\section{Referencias Bibliográficas}

Balza, A. (2011). Complejidad, transdisciplinariedad y transcomplejidad. Los caminos de la nueva ciencia. Caracas: Fondo Editorial Gremial Apunersr.

Bedford, E (1957). Emociones y procedimientos de la sociedad aristotélica ,57pp.

Bourdieu y Wacquant, (1995). Una invircion a la Sociologia Refresiva. ISBN 987-1220-324. Delsur Alte. Solier. Argentina.

Castillo, S (2008). Propuesta pedagógica basada en el constructivismo para el uso óptimo de las tic en la enseñanza. Revista Latinoamericana de Investigación en Matemática Educativa ISSN: 1665-2436.

Leiva, J. (2016). Abriendo caminos de interculturalidad e inclusión en la escuela. Madrid : Dykinson.

Méndez, C. (2012). Convergencia educativa y diversidad cultural en el EEES: desde las aula universitarias multiculturales . Salamanca : Universidad de Salamanca.

Morales, E. (2010). Diversidad Cultural y acceso a la información . México, D.F: Universidad Nacional Autónoma de México.

Ovey, S. (2016). Los 7 hábitos de la gente altamente efectiva (Snapshots). Madrid: Grupo Planeta.

Paredes, E. (2017). Estrategias y herramientas para mejorar la atención de los alumnos en el aula. EDUforics.

Pelacín, M. (2012). La diversidad cultural en el aula: un reto, una oportunidad. CVC Cervantes, 702-707.

Pierre Bourdieu (2007). De las reglas a las estrategias en: Bourdieu Pierre, Cosas Dichas, Barcelona: Editorial Gedisa, págs. 67-82.

Puigdellívol Aiguadé, I. (1998). La educación especial en la escuela integrada: Una perspectiva desde la diversidad. Barcelona, España: Graó . 
Torres Dávila, María Soledad. "Género y discapacidad: más allá del sentido de la maternidad diferente". (2004). https://digitalrepository.unm.edu/abya_yala/347.

UNESCO. (1994). Prinicipios de la declaración de Salamanca . Declaración de Salamanca .

Unesco. (2014). Enfoques estratégicos sobre las TICS en educación en América Latina y el Caribe. Santiago de Chile, Chile: OREALC.

Vivas Garcia, Mireya. La Educacion Emocional: Conceptos funfamentales. Sapiens. Revista Universitaria de investigación 2003, 4 )(2), 0. (fecha de consulta 20 de julio de 2020) ISSN: 1317-5815.

Discapacitadas. Educación y Diversidad. Revista Inter-universitaria de Investigación sobre Discapacidad e Interculturalidad. 


\section{PARA CITAR EL ARTÍCULO INDEXADO.}

García Parrales, S. L., Mego Cubas, C., \& Pazmiño Solys, G. A. (2020). Modelo de enseñanza para la atención inclusiva a las necesidades no asociadas a una discapacidad de los estudiantes de la Unidad Educativa Ciudad de Valencia. ConcienciaDigital, 3(3.1), 155160. https://doi.org/10.33262/concienciadigital.v3i3.1.1377

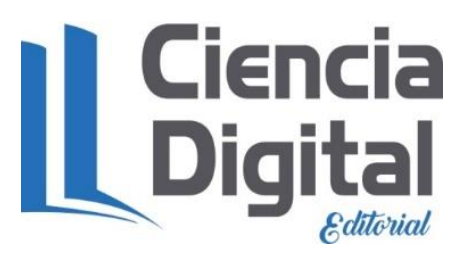

El artículo que se publica es de exclusiva responsabilidad de los autores y no necesariamente reflejan el pensamiento de la Revista Conciencia Digital.

El artículo queda en propiedad de la revista y, por tanto, su publicación parcial y/o total en otro medio tiene que ser autorizado por el director de la Revista Conciencia Digital.
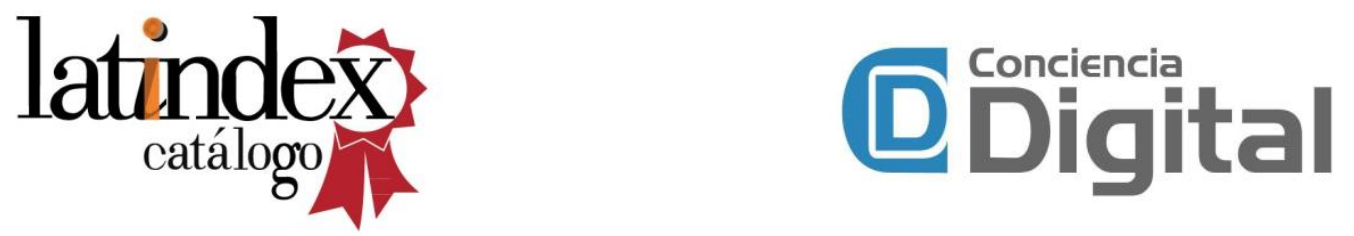